\title{
Testicular volume and elasticity changes in young children with undescended testes
}

\author{
Hyun Joo Shin', Yong Seung Lee², Haesung Yoon', Myung-Joon Kim¹, Sang Won Han², \\ Ha Yan Kim³, Jinae Lee ${ }^{3}$, Mi-Jung Lee ${ }^{1}$
}

${ }^{1}$ Department of Radiology and Research Institute of Radiological Science, Severance Children's Hospital, ${ }^{2}$ Department of Urology and Urological Science Institute, Severance Children's Hospital, ${ }^{3}$ Biostatistics Collaboration Unit, Yonsei University College of Medicine, Seoul, Korea

\begin{abstract}
Aims: To evaluate the differences and changes of testicular volume and elasticity in normal (NL) testes and undescended testes (UDTs) of children using shear wave elastography (SWE). Materials and methods: Testicular ultrasound images from children younger than 60 months old were retrospectively reviewed. Testicular volumes and elasticities were compared between the UDT group and NL group. In patients with unilateral UDT (uUDT), we also compared the values between uUDT and contralateral grossly normal (CGN) testis groups. Results: There were 25 UDTs including 4 bilateral in the UDT group and 54 normal testes in the NL group. While testicular volume was significantly smaller in UDT (vs. NL) and uUDT (vs. CGN) groups, the elasticity was not different. Testicular volume was positively correlated with age in both NL (r=0.474) and CGN $(\mathrm{r}=0.729)$ groups $(\mathrm{p}<0.001)$, while there was no correlation in the UDT group. Testicular stiffness showed negative correlation with age in the NL group $(r=-0.390, p=0.004)$ and positive correlation in the UDT group $(r=0.426$, $p=0.034)$. Conclusions: Instead of increasing volume and decreasing stiffness of normal testes during development of the early 60 months, UDTs exhibited smaller volume and increasing stiffness. The CGN testes of uUDT patients showed increasing volume without stiffness change.
\end{abstract}

Keywords: undescended testes; ultrasonography; elastography; children

\section{Introduction}

Undescended testis (UDT) or cryptorchidism is a condition in which one or both testes is not visible in the scrotum [1]. It is the most common anomaly in boys, encountered in about $2-8 \%$ of full-term and $33 \%$ of preterm boys [1]. Even though UDT incidence decreases to $1-2 \%$ by 3 to 6 months of age due to spontaneous descent, the persistent UDT cases require surgical correc-

Received 10.05.2017 Accepted 12.07.2017

Med Ultrason

2017, Vol. 19, No 4, 380-385

Corresponding author: Mi-Jung Lee, $\mathrm{MD}, \mathrm{PhD}$

Department of Radiology and Research Institute of Radiological Science, Severance Children's Hospital,

Yonsei University College of Medicine,

50-1 Yonsei-ro, Seodaemun-gu, Seoul,

Korea 03722

Phone: 82-2-2228-7400, Fax: 82-2-393-3035

E-mail:mj11213@yuhs.ac tion due to the risk of infertility or developing tumors [13]. A recent general recommendation for the timing of UDT orchiopexy is within the first 18 months of age to prevent early histologic change [3]. However, the actual timing for surgery differs according to the various conditions in many countries $[1,2,4,5]$.

It is well known that UDT has abnormal histopathologic features, including decreased germ-cell counts, spermatogenesis failure, Leydig-cell hypoplasia, and testicular fibrosis [6]. UDT could disturb normal spermatogenesis by delaying transformation of germ cells to adult dark spermatocytes in the first three months of life. Interstitial and tubular damage could also accompany UDT, which could be responsible for the high risk of azoospermia associated with UDT - incidences of 13\% in unilateral cases and $89 \%$ in bilateral cases [7]. Additionally, abnormal anatomical locations for UDTs may increase the risk for malignant transformations that could develop into testicular cancer [7]. Decreased vascularity 
in UDTs has also been demonstrated by histology [8]. However, there are limited tools to evaluate these histopathologic changes during neonatal and early childhood growth in boys.

Imaging studies have traditionally played a limited role in the evaluation of UDTs, and the American Urologic Association guideline mentioned that the imaging modalities rarely assisted in decision making [3]. Invasive testicular biopsy is the only method for evaluating the histopathologic changes of UDTs in children. Therefore, in the last years there were efforts for the detection in the pathologic changes of UDTs using noninvasive imaging modalities. Some previous studies, using Doppler ultrasonography (US) or Superb Microvascular Imaging demonstrated decreased perfusion in UDTs which was compatible with known histologic findings [8,9]. In addition, US elastography is a simple noninvasive method for measuring tissue elasticity [10]. Although a few studies have tried to use this technique in UDTs [11-15], to our knowledge there has not been a study that compared the elasticity differences between normal testes and UDTs in young children preoperatively using shear wave elastography (SWE).

Therefore, the purposes of our study were 1) to evaluate and compare the volume and elasticity of normal testes and UDTs and 2) to evaluate the changes and correlations between these values and the age of the young children.

\section{Materials and methods}

\section{Subjects}

The Institutional Review Board approved this retrospective study and waived informed consent. The testicular US images, including SWE, performed between March 2015 and June 2016 in children less than 60 months old were reviewed. The protocol of our department includes in the routine testicular US, the use of SWE in all children, without consideration of the clinical indication

Children with unilateral or bilateral UDTs were included (UDT group). In children with unilateral UDTs, their UDTs were grouped as unilateral UDT (uUDT) and their contralateral grossly normal and descended testes were grouped as contralateral grossly normal (CGN) testes. Children with both testes descended and without focal lesions or inflammatory changes (testicular swelling, increased vascularisation on Doppler US, or debris in hydrocele) formed the normal (NL) group.

\section{Testicular US including SWE}

Testicular US, including supersonic SWE (Aixplorer; SuperSonic Imagine, Aix-en-Provence, France; software version of 9.2), was performed with a $15-4 \mathrm{MHz}$ linear transducer. One of two experienced pediatric radiologists performed the examinations. First, routine US was performed on both scrotums and inguinal areas while children were in a supine position in order to evaluate the testicular location. When the testis was not visible in the scrotum, the inguinal area or abdomen was examined and its location was recorded. Three-dimensional diameters of the testis were measured and testis volume was automatically calculated in the unit of cc using the ellipsoid volume measurement method; volume $(\mathrm{cc})=0.523 \times$ height $(\mathrm{cm}) \times$ width $(\mathrm{cm}) \times$ depth $(\mathrm{cm})$.

Testicular elasticity were measured by applying minimal pressure from the transducer on the scrotum, in the homogenous parenchyma at the center of each testis, with perpendicular orientation of the probe to the testicular long axis, and care to avoid the mediastinal testis; all measurements were repeated three times and the average value was used for analysis [9]. A round region of interest (ROI) was placed in the central portion of testicular parenchyma on each color-coded map of elasticity after obtaining a homogeneous color without visible artifacts (fig 1). The mean acquisition depth in three measurements was recorded in each testis to evaluate the effect of acquisition depth on the results of elasticity [16]. The mean values of the testicular elasticities within the ROI were automatically provided in each acquisition, using $\mathrm{kPa}$ units, based on Young's modulus.

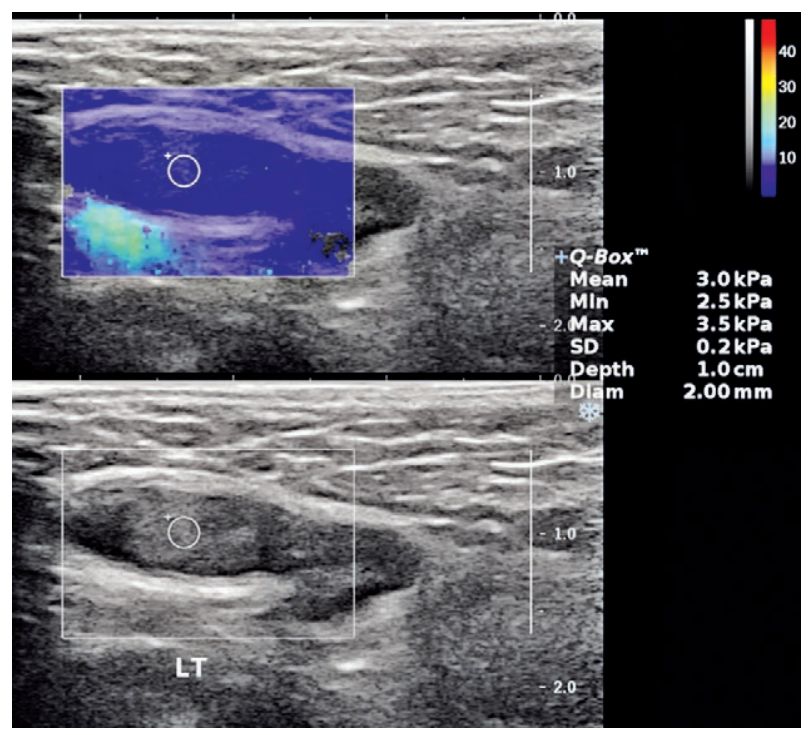

Fig 1. Image from a 6-month-old boy in the unilateral undescended testis (UDT) group. Left UDT was noted in left upper inguinal canal, with volume of $0.2 \mathrm{cc}$. Testicular elasticity was measured using a round region of interest placed on colorcoded map of elasticity without artifacts. The mean elasticity of UDT from three repeated measurements was $3.8 \mathrm{kPa}$. The contralateral normal testis (not shown) was $0.6 \mathrm{cc}$ with mean elasticity of $2.6 \mathrm{kPa}$. 


\section{Statistical analyses}

Statistical analyses were performed using SAS version 9.2 software (SAS, Cary, NC, USA). After a normality test for age and volume, the testicular volumes between the NL and UDT groups, and between the NL and CGN groups were compared using the Mann-Whitney U test. The testicular volumes between the uUDT and CGN groups were compared using the Wilcoxon signed rank test. Testicular elasticities between the groups were compared using the clustered regression test with age adjustment. The correlations between age and volume or testicular elasticity in each group were evaluated using the Spearman correlation test. For these analyses, p-values less than 0.05 were considered statistically significant.

\section{Results}

\section{Patients}

During the study period 24 children less than 60 months underwent testicular US with SWE for suspected UDT. In 3 children, the UDTs were not detected on US. In 21 children, unilateral or bilateral UDTs were found (UDT group) (mean age 12.1 \pm 11.8 months, range 0-43 months). The normal group consisted of 27 children (mean age $15.8 \pm 15.8$ months, range $0-53$ months). There was no child with focal mass or inflammatory change in testis.
In the UDT group we found 25 UDTs: 7 in the right side, 10 in the left, and 4 bilateral. The UDTs were located in the inguinal area in 23 cases and in the lower abdomen in 2 cases. The 17 children with unilateral UDTs had mean age of $12.2 \pm 12.8$ months and age range of 0-43 months.

\section{Evaluation and comparison of testicular volume and elasticity}

The results of testicular volume and elasticity are detailed in Table I. The testicular volume in the UDT group was lower than that of the NL group ( $p<0.001)$. The mean testicular volume of the uUDT group was also lower compared with the CGN group $(\mathrm{p}<0.001)$. The volume of testes in the CGN group was significantly larger than that of the NL group ( $\mathrm{p}=0.019)$.

In SWE, the ROI size was $2-4 \mathrm{~mm}$ and the acquisition depth was $0.5-1.6 \mathrm{~cm}$. The acquisition depths were different between the NL $(0.8 \pm 0.2 \mathrm{~cm})$ and UDT $(1.1 \pm 0.3$ $\mathrm{cm})$ groups $(\mathrm{p}<0.001)$. However, the mean acquisition depth was not different between the uUDT $(1.0 \pm 0.3 \mathrm{~cm})$ and CGN $(0.9 \pm 0.2 \mathrm{~cm})$ groups $(\mathrm{p}=0.104)$. The mean testicular elasticity of the NL group was $3.9 \pm 0.7 \mathrm{kPa}$ (range 2.3-6.2 $\mathrm{kPa}$ ), and that of the UDT group was $4.4 \pm 2.6 \mathrm{kPa}$ (range 1.0-13 kPa). The elasticity of the UDT group was not different from that of the NL group $(\mathrm{p}=0.372)$. The mean testicular elasticity of the uUDT group (4.6 $6 \pm 2.9$ $\mathrm{kPa}$, range $1.0-13 \mathrm{kPa})$ and the CGN group $(3.8 \pm 1.3 \mathrm{kPa}$, range $1.9-7.3 \mathrm{kPa})$ was not different either $(\mathrm{p}=0.263)$.

Table I. Comparison of the normal (NL) and undescended testis (UDT) groups, and unilateral UDT (uUDT) and contralateral grossly normaltestis (CGN) groups.

\begin{tabular}{llll}
\hline & NL group (54 testes of 27 children) & UDT group (25 testes of 21 children) & p-value \\
\hline Age (months) & $15.8 \pm 15.8$ & $12.1 \pm 11.8$ & $0.560^{*}$ \\
Testicular volume $(\mathrm{cc})$ & $0.5 \pm 0.2$ & $0.3 \pm 0.2$ & $<0.001^{*}$ \\
Testicular elasticity $(\mathrm{kPa})$ & $3.9 \pm 0.7$ & $4.4 \pm 2.6$ & $0.372^{* *}$ \\
\hline & \multicolumn{1}{c}{ Unilateral UDT (uUDT) (17 children) } \\
\hline & CGN group & uUDT group & $<0.001^{* * *}$ \\
Testicular volume $(\mathrm{cc})$ & $0.7 \pm 0.3$ & $0.3 \pm 0.2$ & $0.263^{* *}$ \\
Testicular elasticity $(\mathrm{kPa})$ & $3.8 \pm 1.3$ & $4.6 \pm 2.9$ & \\
\hline
\end{tabular}

$\mathrm{NL}$ - normal testes, UDT - undescended testis, uUDT - unilateral UDT, CGN - contralateral grossly normal testis, ${ }^{*}$ Mann - Whitney U test, ** Clustered regression test, ***Wilcoxon signed rank
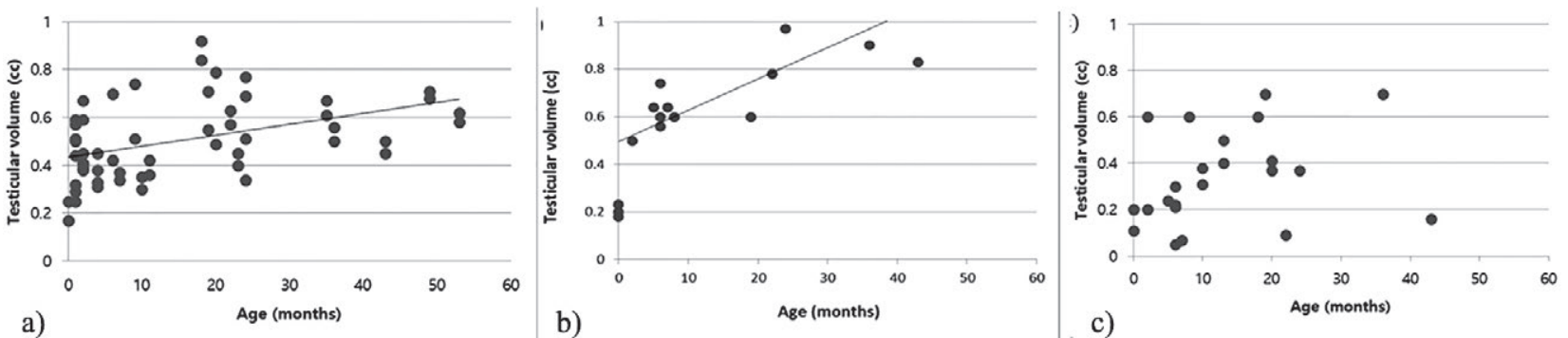

Fig 2. Scatter plots of age and testicular volume in the normal (NL) group (a), contralateral grossly normal (CGN) group from children with unilateral UDTs (b), and UDT groups (c). (a and b) Testicular volume was positively correlated with age (a) in the NL group ( $\mathrm{r}=0.474, \mathrm{p}<0.001)$ and (b) in the CGN group $(\mathrm{r}=0.729, \mathrm{p}<0.001)$. (c) However, testicular volume was not significantly correlated with age in the UDT group $(\mathrm{r}=0.391, \mathrm{p}=0.053)$. 

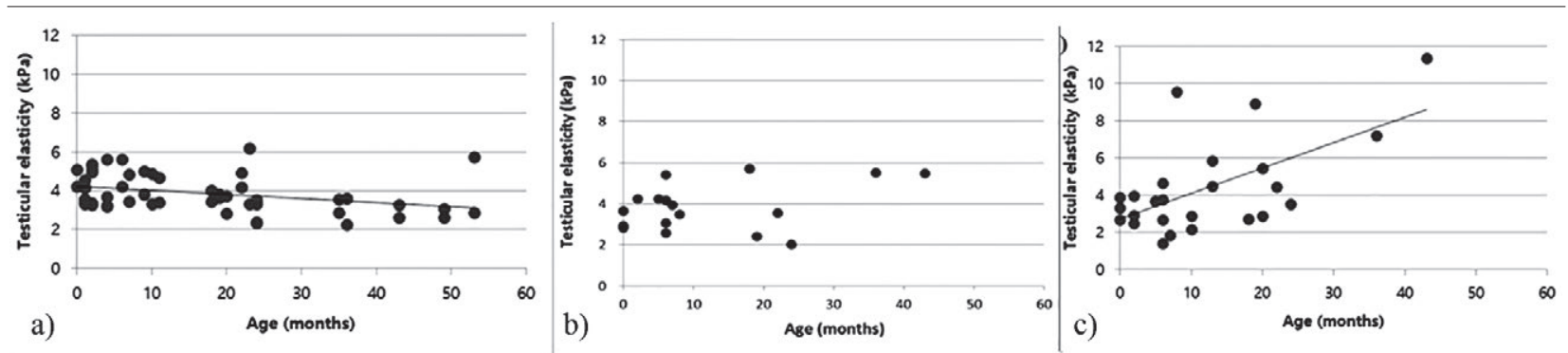

Fig 3. Scatter plots of age and testicular elasticity in the NL group (a), CGN group (b) and UDT group (c). (a) There was a negative correlation between testicular stiffness and age in the NL group $(r=-0.390, p=0.004)$. (b) However, there was no significant correlation between testicular stiffnessand age in the CGN group ( $\mathrm{r}=0.177, \mathrm{p}=0.497)$. (c) Moreover, there was positive correlation between stiffnessand age in the UDT group $(\mathrm{r}=0.426, \mathrm{p}=0.034)$.

\section{Correlation of testicular volume and elasticity with age in each group}

In the NL group, testicular volume was positively correlated with age ( $\mathrm{r}=0.474, \mathrm{p}<0.001$; fig 2$)$. The CGN group also showed positive correlation between testicular volume and age $(\mathrm{r}=0.729, \mathrm{p}<0.001)$. However, testicular volume was not significantly correlated with age in both the UDT group $(\mathrm{r}=0.391, \mathrm{p}=0.053)$ and the uUDT group $(\mathrm{r}=0.268, \mathrm{p}=0.298)$.

In testicular elasticity (fig 3 ), there was a negative correlation between testicular stiffness and age in the NL group ( $\mathrm{r}=-0.390, \mathrm{p}=0.004)$. However, in the CGN group, there was no significant correlation between testicular stiffness and age $(r=0.177, p=0.497)$. Moreover, there was positive correlations between stiffness and age in both the UDT group ( $\mathrm{r}=0.426, \mathrm{p}=0.034)$ and the uUDT group $(\mathrm{r}=0.502, \mathrm{p}=0.040)$.

\section{Discussions}

The first attempt to measure testicular elasticity using US was in 2011, wherein the researchers used testicular sonography, including acoustic radiation force impulse imaging, to measure testicular volume and elasticity in an adult population ( $>20$ years old) [17]. They found that stiffness increased and volume decreased as age increased. Following this study, testicular elastography was used to examine various types of testicular focal lesions, torsion or segmental infarctions [13,18-24]. These studies demonstrated the feasibility of elastography for evaluating elasticity in normal and diseased testes. However, only a few studies usedelastography to evaluate testicular elasticity in children with UDT.

In 2015, strain elastography was used to discriminate cases of UDT from reactive lymph node cases in children and demonstrated that reactive lymph nodes were stiffer than UDTs [11]. A recent study using SWE showed that the mean testicular stiffness was significantly higher in
uUDT than in CGN, without significant volume difference, in children with an age range of 1-18 years [15]. Another study used point SWE for postoperative UDTs, and demonstrated the different stiffness in postoperative UDTs compared to CGN and normal testes [14]. However, they did not evaluate the growth pattern of UDTs. This is the first study to compare testicular elasticity in children with normal and UDTs including CGN testes using SWE preoperatively in considering with growth change.

In our study, mean testicular volume was $0.5 \pm 0.2 \mathrm{cc}$ with a mean elasticity of $3.9 \pm 0.7 \mathrm{kPa}$ in the NL group who were all $<60$ months old. Mean testicular volume was $0.3 \pm 0.2 \mathrm{cc}$ with a mean elasticity of $4.4 \pm 2.6 \mathrm{kPa}$ in the UDT group. Although volume was significantly smaller in the UDT group, stiffness was not different between the two groups. Stiffness can be increased when increasing acquisition depth using linear transducer of Aixplorer [16]. However, the range of mean acquisition depth was small $(0.8-1.1 \mathrm{~cm})$ in our study. The stiffness was still different between uUDT and CGN groups, even though the acquisition depth was not different in this comparison.

Moreover, there was a tendency toward increasing testicular volume and decreasing stiffness with age in normal young children, while the tendency in the UDT group was toward increasing stiffness with age. The reported mean testicular elasticity in adults was $0.9 \mathrm{~m} / \mathrm{sec}$ in the center portion, which converted to $2.4 \mathrm{kPa}$ [25]. The reported mean central testicular elasticity in healthy children with a mean age of 9 years old was $2.5 \mathrm{kPa}$ [13]. In comparison, in our study, mean normal testicular elasticity in children $<5$ years old was $3.9 \mathrm{kPa}$; this finding supports the evidence for decreasing stiffness with age in normal testes of young children. The elasticity change is different in UDT. D'Anastasi et al [17] argued that testicular stiffness increased among aging men and that testis size also decreased in response to increased intertubular fibrosis and testicular atrophy. In old age, testicular 
size decreases due to tubular sclerosis and atrophy [25]. Similar changes have been observed among children with UDT. Histology of several UDTs revealed that Leydig-cell hypoplasia was apparent in the first two months of life, decreased germ-cell count was apparent by four months, and a gradual increase in fibrosis occurred when these conditions were left untreated [6]. These developmental patterns could explain why higher stiffness was observed in UDTs, which resembled similar patterns seen in the gradually atrophied testes of elderly men.

Kuijper et al [26] studied testicular volumes in 344 children between 0-6 years old using US and showed differences in mean testicular volume according to minipuberty in the first year of life. They also found that mean testicular volume increased from $0.27 \mathrm{cc}$ to $0.44 \mathrm{cc}$ in the first five months of life, followed by a decrease, and ultimately stabilizing at $0.31 \mathrm{cc}$. Mean testicular volume was relatively larger and tended to increase in our study group; however, we could not compare testicular volume in each age group according to mini-puberty because of the small number of infants in our study. In addition, testicular volume and age correlation in the UDT group was close to being significant with $\mathrm{p}=0.053$, even though the result in the uUDT group was not significant $(\mathrm{p}=0.298)$. Additional studies with more patients are needed to validate the volume change.

Interestingly, CGN testes in children with uUDTs were affected in their volume and elasticity by showing differences from NL testes of age-adjusted young children in our study. The volumes of CGN testes were larger than that of uUDTs and NL testes. Even though the testicular volume increased as increasing age, the stiffness was not correlated with age in the CGN group. Previous studies also demonstrated that the UDTs could affect contralateral descended testes, causing pathological changes and increased risk of malignancy than normal testes $[27,28]$. Our results support the difference of the contralateral descended testes showing lack of normal elasticity change. Further studies are required to evaluate the relationship between the elasticity difference and histopathologic abnormality in the CGN testes of uUDT patients.

This study has several limitations. Firstly, because this was a retrospective study, the age distribution was uneven and we had an uneven number of children in the study groups, with fewer in the UDT group than in the NL group. Also, there were fewer children in the older age categories. However, mean age was not statistically different between the two groups. Secondly, the direct comparison between histopathology and elastography results of the testes was not available in this study, because biopsy was not a routine procedure for UDTs. Thirdly, we did not assess inter-observer variability for measuring testicular elasticity. Instead, we tried to measure elasticity using a homogeneous color map without visual artifacts, and repeated measurements three times to reduce variability. At last, two intra-abdominal testes were included in the UDT group. Because of the small number, it was hard to know the effect of environment of the testicular location on testicular stiffness. Further studies for assessing elasticity differences according to the location of UDTs are required in a large study group.

\section{Conclusions}

Instead of increasing volume and decreasing stiffness of normal testes, UDTs exhibited smaller volume and increasing stiffness during early growth under 60 months of age. In addition, the volume and stiffness changes of the contralateral descended testes were different from those of the bilaterally normal testes. Therefore, not only UDTs but also contralateral descended testes need to be evaluated in young children including SWE evaluation.

\section{Conflict of interest: none}

\section{References}

1. Niedzielski JK, Oszukowska E, Slowikowska-Hilczer J. Undescended testis - current trends and guidelines: a review of the literature. Arch Med Sci 2016;12:667-677.

2. Schneuer FJ, Holland AJ, Pereira G, Jamieson S, Bower C, Nassar N. Age at Surgery and Outcomes of an Undescended Testis. Pediatrics 2016;137:e20152768.

3. Kolon TF, Herndon CD, Baker LA, et al. Evaluation and treatment of cryptorchidism: AUA guideline. J Urol 2014;192:337-345.

4. Hrivatakis G, Astfalk W, Schmidt A, et al. The timing of surgery for undescended testis - a retrospective multicenter analysis. Dtsch Arztebl Int 2014;111:649-657.

5. Hensel KO, Caspers T, Jenke AC, Schuler E, Wirth S. Operative management of cryptorchidism: guidelines and reality--a 10-year observational analysis of 3587 cases. BMC Pediatr 2015;15:116.

6. Mechlin CW, Kogan BA. What lessons can be learned from testicular histology in undescended testes? Transl Androl Urol 2014;3:365-369.

7. Fawzy F, Hussein A, Eid MM, El Kashash AM, Salem HK. Cryptorchidism and Fertility. Clin Med Insights Reprod Health 2015;9:39-43.

8. Atilla MK, Sargin H, Yilmaz Y, Odabas O, Keskin A, Aydin $\mathrm{S}$. Undescended testes in adults: clinical significance of resistive index values of the testicular artery measured by Doppler ultrasound as a predictor of testicular histology. J Urol 1997;158:841-843.9. Lee YS, Kim MJ, Han SW, et al. Superb microvascular imaging for the detection of pa- 
renchymal perfusion in normal and undescended testes in young children. Eur J Radiol 2016;85:649-656.

9. Jeong WK, Lim HK, Lee HK, Jo JM, Kim Y. Principles and clinical application of ultrasound elastography for diffuse liver disease. Ultrasonography 2014;33:149-160.

10. Agladioglu K, Herek D, Herek O, Agladioglu SY, Corduk N, Ozhan B. Can ultrasound elastography be used as a new technique in the differentiation of undescended testes and reactive lymph nodes in children? Clin Radiol 2015;70:1269-1275.

11. Zhang X, Lv F, Tang J. Shear wave elastography (SWE) is reliable method for testicular spermatogenesis evaluation after torsion. Int J Clin Exp Med 2015;8:7089-7097.

12. Sun Z, Xie M, Xiang F, et al. Utility of Real-Time Shear Wave Elastography in the Assessment of Testicular Torsion. PLoS One 2015;10:e0138523.

13. Hattapoglu S, Goya C, Arslan S, et al. Evaluation of postoperative undescended testicles using point shear wave elastography in children. Ultrasonics 2016;72:191-194.

14. Ucar AK, Alis D, Samanci C, et al. A preliminary study of shear wave elastography for the evaluation of unilateral palpable undescended testes. Eur J Radiol 2017;86:248-251.

15. Shin HJ, Kim MJ, Kim HY, Roh YH, Lee MJ. Comparison of shear wave velocities on ultrasound elastography between different machines, transducers, and acquisition depths: a phantom study. Eur Radiol 2016;26:3361-3367.

16. D'Anastasi M, Schneevoigt BS, Trottmann M, et al. Acoustic radiation force impulse imaging of the testes: a preliminary experience. Clin Hemorheol Microcirc 2011;49:105-114.

17. Patel K, Sellars ME, Clarke JL, Sidhu PS. Features of testicular epidermoid cysts on contrast-enhanced sonography and real-time tissue elastography. J Ultrasound Med 2012;31:115-122.

18. Lock G, Schroder C, Schmidt C, Anheuser P, Loening T, Dieckmann KP. Contrast-enhanced ultrasound and realtime elastography for the diagnosis of benign Leydig cell tumors of the testis - a single center report on 13 cases. Ultraschall Med 2014;35:534-539.

19. Jedrzejewski G, Ben-Skowronek I, Wozniak MM, Brodzisz A, Budzynska E, Wieczorek AP. Testicular adrenal rest tumors in boys with congenital adrenal hyperplasia: 3D US and elastography--do we get more information for diagnosis and monitoring? J Pediatr Urol 2013;9:1032-1037.

20. Pastore AL, Palleschi G, Maceroni P, et al. Correlation between semiquantitative sonoelastography and immunohistochemistry in the evaluation of testicular focal lesions. Cancer Imaging 2014;14:29.

21. Yusuf G, Konstantatou E, Sellars ME, Huang DY, Sidhu PS. Multiparametric Sonography of Testicular Hematomas: Features on Grayscale, Color Doppler, and Contrast-Enhanced Sonography and Strain Elastography. J Ultrasound Med 2015;34:1319-1328.

22. Zeng B, Chen F, Qiu S, et al. Application of Quasistatic U1trasound Elastography for Examination of Scrotal Lesions. J Ultrasound Med 2016;35:253-261.

23. Kantarci F, Cebi Olgun D, Mihmanli I. Shear-wave elastography of segmental infarction of the testis. Korean J Radiol 2012;13:820-822.

24. Trottmann M, Marcon J, D'Anastasi M, et al. Shearwave elastography of the testis in the healthy man - determination of standard values. Clin Hemorheol Microcirc 2016;62:273-281.

25. Kuijper EA, van Kooten J, Verbeke JI, van Rooijen M, Lambalk CB. Ultrasonographically measured testicular volumes in 0- to 6-year-old boys. Hum Reprod 2008;23:792-796.

26. Verkauskas G, Malcius D, Eidukaite A, et al. Prospective study of histological and endocrine parameters of gonadal function in boys with cryptorchidism. J Pediatr Urol 2016;12:238.e1-e6.

27. Chung JM, Lee SD. Individualized Treatment Guidelines for Postpubertal Cryptorchidism. World J Mens Health 2015;33:161-166. 